\title{
On Solving Singular Integral Equations via a Hyperbolic Tangent Quadrature Rule
}

\author{
By Ezio Venturino*
}

\begin{abstract}
We propose a scheme for solving singular integral equations based on a "hyperbolic tangent" quadrature rule. The integral equation is reduced to a system of linear equations, after quadrature and collocation. The matrix of the system is shown to be nonsingular for every choice of the number of quadrature nodes by producing a lower bound for its determinant.
\end{abstract}

1. Introduction. Various methods have been discussed in the literature for solving singular integral equations. In this paper, we examine a method which utilizes the quadrature formulas proposed by F. Stenger [4] and show the nonsingularity of the system of linear algebraic equations which is obtained from discretization of the original equation. The formulas proposed in [4] for singular integrals are based on the use of the Whittaker cardinal function [2], [8]. They have been applied in [7] to singular integral equations using a Galerkin-type approximation. Both the method and the quadrature schemes were designed to handle problems whose solutions may have singularities at the endpoints of the interval, the exact nature of which is difficult to determine. This is the case in almost all the Cauchy singular integral equations arising in the applications.

The scheme we propose to use for solving the singular integral equation makes use of the "hyperbolic tangent" quadrature rule. After quadrature and collocation at two distinct sets of nodes, the integral equation is reduced to a system of linear algebraic equations. Owing to the special form of the quadrature and collocation nodes, a new set of knots is generated, at which the unknown function is finally evaluated. The matrix of the system is shown to be nonsingular. Two proofs are provided, the second of which is geometric and allows the computation of a lower bound on the determinant.

2. Preliminaries. Our concern here is with the following singular integral equation,

$$
(1 / \pi) f_{-1}^{1}[g(s) d s /(s-x)]=f(x), \quad-1<x<1,
$$

subject to the normalization condition

$$
\int_{-1}^{1} g(x) d x=C
$$

Received June 13, 1985.

1980 Mathematics Subject Classification. Primary 45E05.

* Permanent address. Dipartimento di Matematica, Università di Torino, via Carlo Alberto 10, 10123 Torino, Italy. 
which, as it is well known, ensures uniqueness of the solution. The quadrature formulas and the error bounds we need are given here without proofs, which can be found in [4]:

$$
\begin{aligned}
& \left|\int_{-1}^{1} g(x) d x-h \sum_{k=-N}^{N} 2 E(1+E)^{-2} g((E-1) /(E+1))\right| \\
& \quad \leqslant C_{2} \exp (-\pi \sqrt{\alpha N}) / \alpha
\end{aligned}
$$

with

$$
\begin{gathered}
E=\exp (k h), \quad h=\pi / \sqrt{\alpha N} \\
|g(x)| \leqslant C^{2}\left(1-x^{2}\right)^{\alpha} \\
\left|(h / 2 \pi) \sum_{k=-M}^{M} \frac{1-x_{k}^{2}}{x_{k}\left(1+x x_{k}\right)} g\left(\frac{x+x_{k}}{1+x x_{k}}\right)-(1 / \pi) f_{-1}^{1} g(t) /(t-x) d t\right| \\
\leqslant C_{3}(x) \exp (-\pi \sqrt{\beta M}) / \beta, \quad-1<x<1,
\end{gathered}
$$

with

$$
h=\pi / \sqrt{\beta M}, \quad|g(x)|<C^{3}\left(1-x^{2}\right)^{\beta-1}, \quad x_{k}=\tanh ((k+1 / 2) h / 2) .
$$

There are two ways of applying these quadrature formulas, corresponding to the cases in which we choose either a different or the same number of quadrature nodes. These choices lead to two different linear algebraic systems. We obtain these systems by replacing the integral by a quadrature formula, this operation leading to a functional equation. Then, we discretize it by collocating at a set of knots. The main feature in using the above-mentioned quadrature formula is that it leads, together with a proper choice for the set of collocation nodes, to a third set of nodes, distinct from the quadrature and the collocation ones. Let us call the last set of knots the "evaluation nodes". Indeed, it is at this set of nodes that the unknown function will be finally calculated, by solving the algebraic system. We turn now to the discussion of how the matrices are constructed.

The First Approach. We use (2.2) with $M=2 N$. For the principal value integral we thus have the approximation

$$
(1 / \pi) f_{-1}^{1} g(t) /(t-x) d t=(h / 2 \pi) \sum_{k=-2 N}^{2 N} \frac{1-x_{k}^{2}}{x_{k}\left(1+x x_{k}\right)} g\left(\frac{x+x_{k}}{1+x x_{k}}\right)
$$

with

$$
h=\pi / \sqrt{2 \beta N}, \quad|g(x)| \leqslant\left(1-x^{2}\right)^{\beta-1},
$$

while for the normalization condition we get

$$
\int_{-1}^{1} g(x) d x=(h / 2) \sum_{k=-N}^{N} \operatorname{sech}^{2}(k h / 2) g(\tanh (k h / 2)) .
$$

Here,

$$
h=\pi / \sqrt{\alpha N}, \quad|g(x)| \leqslant\left(1-x^{2}\right)^{\alpha-1} .
$$


It is clear that in order for the $h$ 's appearing in the two equations to be the same, the choice $\alpha=2 \beta$ has to be made. The conditions on the growth of the function will still be satisfied. Next, we would like to remove the singular behavior of the function $g$ near the endpoints. We thus set

$$
g(t)=F(t) /\left(1-t^{2}\right)
$$

with $F(t)$ being the new unknown function. From Eqs. (2.4) and (2.3) we obtain

$$
\begin{gathered}
(h / 2) \sum_{k=-N}^{N} \operatorname{sech}^{2}(k h / 2) /\left[1-\tanh ^{2}(k h / 2)\right] F(\tanh (k h / 2))=C, \\
h /(2 \pi) \sum_{k=-2 N}^{2 N} \frac{1-x_{k}^{2}}{x_{k}\left(1+x x_{k}\right)} F\left(\frac{x+x_{k}}{1+x x_{k}}\right) /\left[1-\left(\frac{x+x_{k}}{1+x x_{k}}\right)^{2}\right]=f(x) .
\end{gathered}
$$

The normalization condition reduces to

$$
(h / 2) \sum_{k=-N}^{N} F(\tanh (k h / 2))=C,
$$

while the second equation can be rewritten as

$$
h /(2 \pi) \sum_{k=-2 N}^{2 N}\left(x+1 / x_{k}\right) F\left[\left(x+x_{k}\right) /\left(1+x x_{k}\right)\right]=f(x)\left(1-x^{2}\right) .
$$

We now proceed to collocate the above equations at a suitably chosen set of nodes, namely

$$
t_{j}=\tanh ((j-1 / 2) h / 2), \quad j=-N+1, \ldots, N .
$$

This choice has the nice property of reducing the "evaluation" nodes for the unknown function $F$ to a set which includes the unknowns appearing in the normalization condition. The resulting equations are

$$
\begin{aligned}
h /(2 \pi) \sum_{k=-2 N}^{2 N}\left(t_{j}+1 / x_{k}\right) F[\tanh (k+j) h / 2]=f\left(t_{j}\right)\left(1-t_{j}^{2}\right), & \\
j & =-N+1, \ldots, N .
\end{aligned}
$$

Relabeling the unknowns, and denoting by $s_{k}, k=-N, \ldots, N$, the "evaluation nodes", we have the following form for the system

$$
\begin{gathered}
\sum_{l=-N}^{N} F\left(s_{l}\right)=2 C / h, \\
\sum_{l=-2 N+j}^{2 N+j}\left(t_{j}+1 / x_{l-j}\right) F\left(s_{l}\right)=2 \pi f\left(t_{j}\right)\left(1-t_{j}^{2}\right) / h, \quad j=-N+1, \ldots, N,
\end{gathered}
$$

where $s_{l}=\tanh (\operatorname{lh} / 2)$. It is clear that in the above system we have more unknowns than equations. It is, however, possible to reduce (2.5) to a square system by the following observation. For $N$ large enough the nodes $s_{l}, l>N$, will cluster around the point 1 , where the function $F(x)$ has a zero. It is then reasonable to set the unknowns $F\left(s_{l}\right)$ for $l>N$ to be identically zero. We then obtain the final form of 
the system as

$$
\begin{gathered}
\sum_{l=-N}^{N} F\left(s_{l}\right)=2 C / h, \\
\sum_{l=-N}^{N}\left(t_{j}+1 / x_{l-j}\right) F\left(s_{l}\right)=2 \pi f\left(t_{j}\right)\left(1-t_{j}^{2}\right) / h, \quad j=-N+1, \ldots, N .
\end{gathered}
$$

The Second Approach. Here we use the same number of quadrature nodes in the two formulas for the normalization condition and the principal value integral, as well as the same parameter specifying the upper bound on the growth of the unknown function. In other words, we set $M=N$ and $\beta=\alpha$ in (2.2). Proceeding similarly as above, we obtain the following form for the system,

$$
\begin{gathered}
\sum_{l=-N}^{N} F\left(s_{l}\right)=2 C / h, \\
\sum_{l=-N}^{\max (-N+j,-N)}\left(1+t_{j}\right) F\left(s_{l}\right)+\sum_{l=\min (N, N+j)}^{N}\left(1+t_{j}\right) F\left(s_{l}\right) \\
+\sum_{l=\max (-N+j,-N)}^{\min (N, N+j)}\left(t_{j}+1 / x_{l-j}\right) F\left(s_{l}\right) \\
=2 \pi f\left(t_{j}\right)\left(1-t_{j}^{2}\right) / h, \quad j=-N+1, \ldots, N .
\end{gathered}
$$

The problem is to see for which values of the parameter $h$ the systems (2.6) and (2.7) are nonsingular. We remark that $h$ depends on the number of quadrature nodes and on the parameter $\alpha$. The result of the next section shows that no conditions on $h$ are necessary in order to ensure the invertibility of the matrices. The systems are also solvable for any choice of the number of quadrature nodes, i.e., for any dimension the systems have.

\section{Proof of Nonsingularity.}

First Method. Let the matrix of the system (2.6) be denoted by $A$. We have

$$
\begin{gathered}
A_{1 j}=1, \quad j=1, \ldots, 2 N+1, \\
A_{i j}=t_{i-N-1}+1 / x_{j-i}, \quad i=2, \ldots, 2 N+1, j=1, \ldots, 2 N+1 .
\end{gathered}
$$

Let $A^{*}$ denote the transpose of $A$. By subtracting $\left(1+t_{j-N-1}\right) \operatorname{col}(1)$ from $\operatorname{col}(j)$ of $A^{*}$, for $j=2, \ldots, 2 N+1$, we obtain the matrix $B^{*}=\left[\beta_{i j}\right]$, with

$$
\begin{gathered}
\beta_{i 1}=1, \quad \rho=\exp (h), \\
\beta_{i j}=2 \rho^{j} /\left(\rho^{i+1 / 2}-\rho^{j}\right), \quad i=1,2, \ldots, 2 N+1, j=2,3, \ldots, 2 N+1 .
\end{gathered}
$$

If $\operatorname{det} B^{*}=0$, there exist constants $C_{1}, \ldots, C_{2 N+1}$ not all zero such that

$$
C_{1}+\sum_{j=2}^{2 N+1} 2 \rho^{j} C_{j} /\left(\rho^{i+1 / 2}-\rho^{j}\right)=0, \quad i=1, \ldots, 2 N+1 .
$$

Now let

$$
\phi(x)=\prod_{j=2}^{2 N+1}\left(x-\rho^{j}\right)
$$


and define the polynomial $p(x)$ of degree $\leqslant 2 N$ by

$$
p(x)=C_{1} \phi(x)+\sum_{j=2}^{2 N+1} 2 \rho^{j} C_{j} \phi(x) /\left(x-\rho^{j}\right) .
$$

Since $p$ vanishes at the $2 N+1$ distinct points $x=\rho^{i+1 / 2}, i=1, \ldots, 2 N+1$, it is identically zero. But $\phi\left(\rho^{i+1 / 2}\right) \neq 0$, hence $C_{j}=0$ for $j=1, \ldots, 2 N+1$, which is a contradiction.

Second Method. We just outline here this approach; for further details the reader is referred to [9]. It is possible to find a nonsingular matrix $B$ such that $B A=H_{2 N+1}$ and

$$
\begin{array}{ll}
H_{1 j}=1, & j=1, \ldots, 2 N+1, \\
H_{i j}=a_{j-i}, & 2 \leqslant i \leqslant j \leqslant 2 N+1, \\
H_{i j}=-a_{i-j-1}, & 1 \leqslant j<i \leqslant 2 N+1, \\
a_{j}=1 / x_{j}=\operatorname{coth}((j+1 / 2) h / 2) .
\end{array}
$$

Thus, $A$ is nonsingular if and only if $H=H_{2 N+1}$ is. The argument for showing the invertibility of $H$ can be summarized as follows. Disregarding the first row, we look at the columns of $H$ as being vectors in the $2 N$-dimensional Euclidean space. Then, $H$ is nonsingular if and only if the simplex of these vectors has nonzero volume. By inserting in it another one with nonzero volume, we obtain a lower bound on the determinant of the matrix $H$.

We begin by proving the

Lemma. Given the closed sets $X, Y$ in the Euclidean space $E_{n}$, for any projection $P: E_{n} \rightarrow E_{n}$ we have $P(X) \subset P(Y)$ if and only if $X \subset Y$.

Proof. (Only if). Suppose by contradiction that for every projection $P, P(X) \subset$ $P(Y)$ and there is $z \in X$ such that $z \notin Y$. Since $Y$ is closed, we can apply the principle of separating hyperplanes. There is then

$$
\pi=\left\{t \in E_{n}:\langle c, t\rangle=\lambda, \lambda=\langle c, z\rangle\right\} \subset E_{n}
$$

such that for every $y \in Y$ we have $\langle c, y\rangle<\lambda$.

Let $C$ denote the linear subspace spanned by the vector $c, C=[c]$, and $P_{C}$ the projection on it. The above formulas translate into $P_{C}(z)=\lambda$ and $P_{C}(y)<\lambda$ for every $y \in Y$. It follows that $P_{C}(z) \notin P_{C}(Y)$. Contradiction.

Define the simplex $S_{2 N+1}$ by removing the first row of $H_{2 N+1}$. Also define the matrix of the signs of $H_{2 N+1}$, i.e.,

$$
V_{i j}=\left\{\begin{array}{cc}
+1 & \text { for } j \geqslant i \\
-1 & \text { for } j<i
\end{array}\right.
$$

Let $T_{2 N+1}$ be the simplex spanned by the columns of $V_{2 N+1}$, once the first row has been removed. We have

$$
\begin{aligned}
& \operatorname{det}\left(H_{2 N+1}\right)=(2 N+1) \text { volume }\left(S_{2 N+1}\right), \\
& \operatorname{det}\left(V_{2 N+1}\right)=(2 N+1) \text { volume }\left(T_{2 N+1}\right) .
\end{aligned}
$$

Consider now a direction in $E_{2 N}$, given by the vector $p=\left(p_{1}, \ldots, p_{2 N}\right)$. The next step consists in calculating the projections of the vertices $s_{i}$ and $t_{i}$ of the simplices $S_{2 N+1}$ and $T_{2 N+1}, i=1, \ldots, 2 N+1$, along the aforementioned direction. Since 
these are represented by two sets of $2 N+1$ scalars, the minimum and the maximum in each set are well defined, though they might not be unique. Suppose that for some $k, 0 \leqslant k \leqslant 2 N$, we have

$$
\left\langle p, t_{k+1}\right\rangle=\sum_{i=1}^{k} p_{i}-\sum_{i=k+1}^{2 N} p_{i}=\min _{1 \leqslant l \leqslant 2 N+1}\left\langle p, t_{l}\right\rangle .
$$

It is possible to show that for the corresponding vertex of $S_{2 N+1}$ the following inequalities hold,

$$
\begin{aligned}
\left\langle p, s_{k+1}\right\rangle & \leqslant\left\langle p, t_{k+1}\right\rangle, \\
\left\langle p, s_{j}\right\rangle \geqslant\left\langle p, t_{j}\right\rangle & =\max _{1 \leqslant l \leqslant 2 N+1}\left\langle p, t_{l}\right\rangle .
\end{aligned}
$$

Consider now $x \in T_{2 N+1}$. From (3.3), (3.4) we easily obtain, for any projection $P$ onto a one-dimensional subspace, $P(x) \in\left[P\left(s_{k+1}\right), P\left(s_{j}\right)\right] \subset \mathbf{R}^{\mathbf{1}}$. Given the arbitrariness of $x$ and $P$, there follows $P\left(T_{2 N+1}\right) \subset P\left(S_{2 N+1}\right)$, and from the lemma, $T_{2 N+1} \subset S_{2 N+1}$, which in turn yields

$$
\operatorname{Volume}\left(S_{2 N+1}\right)>\operatorname{Volume}\left(T_{2 N+1}\right)=2^{2 N} /(2 N+1) \text {. }
$$

Hence, the final estimates are obtained,

$$
2^{2 N} \leqslant \operatorname{det} H_{2 N+1} \leqslant(2 N+1)\left(2 a_{0}\right)^{2 N} .
$$

We remark that the proof has been given for the matrix corresponding to the first approach. The second matrix differs from $H_{2 N+1}$ because it has a band of terms involving hyperbolic cotangents, while the remaining ones are 1's. In the above procedure, however, this involves only minor modifications, actually only simplifications. It is thus possible also for this case to derive the bounds given in (3.5).

4. Numerical Results. We describe here a few applications of the method presented in the preceding discussion. There are several ways of implementing the numerical schemes. Since the matrix of the system is generated by the program, formulation (2.6) is slightly easier than (2.7), even though the latter is certainly faster. Indeed, it requires a smaller number of operations. However, the savings in execution time should not be very large, because the major work done by the computer is the Gaussian elimination. Also, instead of simply setting the extra unknowns to be zero, as we did in Section 2, we could plainly make them all equal to the two extremal values, namely $F\left(s_{N}\right)$ and $F\left(s_{-N}\right)$. This procedure involves changes in the values of the first and last columns of the matrix. The differences observed in some experiments are small. There appears to be some advantage for the first procedure in some cases. In other cases, or in some other part of the interval, the second procedure seems to be better. These experiments were performed in Example 3.

Example 1. We consider the following singular integral equation,

$$
(1 / \pi) f_{-1}^{1} g(t) /(t-x) d t=0, \quad-1<x<1,
$$

subject to the normalization condition

$$
(1 / \pi) \int_{-1}^{1} g(t) d t=1
$$




$\begin{array}{cc}\text { NABLE 1 } \\ \text { Nodes } & \text { Absolute error } \\ & \\ 0.00000000 & 5.842000 \mathrm{E}-9 \\ 0.45954112 & 4.343400 \mathrm{E}-9 \\ 0.75883331 & 6.618000 \mathrm{E}-10 \\ 0.90335937 & 4.258700 \mathrm{E}-9 \\ 0.96309154 & 1.067750 \mathrm{E}-8 \\ 0.98617234 & 1.989390 \mathrm{E}-8 \\ 0.99485731 & 3.414630 \mathrm{E}-8 \\ 0.99809260 & 5.700450 \mathrm{E}-8 \\ 0.99929328 & 9.422113 \mathrm{E}-8 \\ 0.99973824 & 1.551690 \mathrm{E}-7 \\ 0.99990306 & 2.552007 \mathrm{E}-7 \\ 0.99996410 & 4.195225 \mathrm{E}-7 \\ 0.99998670 & 6.895757 \mathrm{E}-7 \\ 0.99999507 & 1.133623 \mathrm{E}-6 \\ 0.99999817 & 1.864608 \mathrm{E}-6 \\ 0.99999932 & 3.071568 \mathrm{E}-6 \\ 0.99999975 & 5.080657 \mathrm{E}-6 \\ 0.99999990 & 8.499683 \mathrm{E}-6 \\ 0.99999996 & 1.468613 \mathrm{E}-5 \\ 0.99999998 & 2.809432 \mathrm{E}-5 \\ 0.99999999 & 8.648739 \mathrm{E}-5\end{array}$

The analytic solution is $g(t)=1 / \sqrt{1-t^{2}}$, from which we have $F(t)=\sqrt{1-t^{2}}$. Selected values obtained for the absolute errors are shown in Table 1. It appears that the convergence for the error at the nodes is fast, and there is good agreement between computed and analytical solutions.

Example 2. Here we consider the singular integral equation

$$
\begin{aligned}
(1 / \pi) f_{-1}^{1} g(t) & /(t-x) d t \\
& =\left[x / \sqrt{1-x^{2}}\right] \ln \left|\left[1-x^{2}+\sqrt{1-x^{2}}\right] /\left[1-x^{2}-\sqrt{1-x^{2}}\right]\right|,
\end{aligned}
$$

together with the normalization condition $\int_{-1}^{1} g(t) d t=2$. Again, the analytical solution is known in closed form, $g(t)=|t| / \sqrt{1-t^{2}}$. It is again easily seen that the error at the endpoint +1 gets smaller with an increase in the number of nodes. Working in single precision, we obtained the value zero for the computed solution, corresponding to the analytical solution $F(1)$, by considering a collocation scheme with only 22 nodes; see Table 2.

\section{TABLE 2}

\begin{tabular}{lll}
\multicolumn{1}{c}{ Nodes } & Absolute error & $N$ \\
0.99999999 & $1.218818 \mathrm{E}-4$ & 20 \\
0.99999999 & $1.216840 \mathrm{E}-4$ & 21 \\
1.0000000 & $1.931529 \mathrm{E}-7$ & 21 \\
0.99999999 & $1.218726 \mathrm{E}-4$ & 22 \\
1.0000000 & less than $1 \mathrm{E}-8$ & 22
\end{tabular}


Example 3. As a final example, the following singular integral equation is proposed,

$$
(1 / \pi) f_{-1}^{1} g(t) /(t-x) d t=U_{m-1}(x), \quad m=1,2, \ldots,
$$

subject to $(1 / \pi) \int_{-1}^{1} g(t) d t=0$. The solution is given by

$$
g(t)=T_{m}(t) / \sqrt{1-t^{2}}, \quad m=1,2, \ldots,
$$

where $T_{m}(t)$ and $U_{m-1}(t)$ are the Chebyshev polynomials of first and second kind, respectively. In this case, the solution throughout the interval of integration was calculated. Since the right-hand side of the integral equation is a polynomial of low degree, the error at the evaluation knots is very well behaved, being of the order of .001 or better. Some results are given in Table 3 .

TABLE 3

The degree of the Chebyshev polynomial is $m=4$.

$\begin{array}{cc}\text { Nodes } & \text { Absolute error } \\ 0.00000000 & 7.091795 \mathrm{E}-3 \\ 0.60594050 & 5.340605 \mathrm{E}-3 \\ 0.88641969 & 2.910557 \mathrm{E}-3 \\ 0.97088225 & 1.465757 \mathrm{E}-3 \\ 0.99277583 & 7.225247 \mathrm{E}-4 \\ 0.99822251 & 3.450673 \mathrm{E}-4 \\ 0.99956355 & 1.443033 \mathrm{E}-4 \\ 0.99989288 & 1.729825 \mathrm{E}-5 \\ 0.99997371 & 1.037775 \mathrm{E}-4 \\ 0.99999355 & 3.057750 \mathrm{E}-4 \\ 0.99999841 & 1.107761 \mathrm{E}-3\end{array}$

The degree of the Chebyshev polynomial is $m=8$.

$\begin{array}{cc}\text { Nodes } & \text { Absolute error } \\ 0.00000000 & 1.961645 E-2 \\ 0.45954112 & 1.602601 E-2 \\ 0.75883331 & 1.016630 E-2 \\ 0.90335937 & 6.085182 E-3 \\ 0.96309154 & 3.654146 E-3 \\ 0.98617234 & 2.210092 E-3 \\ 0.99485731 & 1.341594 E-3 \\ 0.99809260 & 8.155942 E-4 \\ 0.99929328 & 4.960732 E-4 \\ 0.99973824 & 3.017339 E-4 \\ 0.99990306 & 1.834430 E-4 \\ 0.99996410 & 1.113656 E-4 \\ 0.99998670 & 6.733893 E-5 \\ 0.99999507 & 4.027224 E-5 \\ 0.99999817 & 2.334706 E-5 \\ 0.99999932 & 1.229572 E-5 \\ 0.99999975 & 4.312979 E-6 \\ 0.99999990 & 2.712145 E-6 \\ 0.99999996 & 1.103880 E-5 \\ 0.99999998 & 2.562212 E-5 \\ 0.99999999 & 8.406757 E-5\end{array}$


A few final comments are in order. The choice for the quadrature nodes implies that they cluster around the endpoints of the interval, where they provide good accuracy. However, the behavior of the solution in the middle of the interval tends to be missed. To increase the number of nodes in order to diminish the length of the subinterval closest to the origin, leads to further complications, as is illustrated by the following computation for $\alpha=.5$. To obtain $x_{0}=.1$ we need $h=.40134$, which corresponds to $N=122$, but to get $x_{0}=.01$, we need $h=.0400$ and $N=12336$. Also, in some cases of oscillatory solutions, the numerical results become poorer than the ones presented here. This is due to the fact that the error bounds appearing in (2.1) become large in these situations.

Acknowledgment. The author thanks Professor R. P. Srivastav for his guidance in this study. This research has been partially supported by a grant of the Italian Consiglio Nazionale delle Ricerche. The author also thanks the referee for useful comments and for providing the first proof in Section 3.

Department of Applied Mathematics and Statistics

SUNY at Stony Brook

Stony Brook, New York 11794

1. F. R. Gantmacher, The Theory of Matrices, Chelsea, New York, 1959.

2. J. MCNAmeE, F. Stenger \& E. L. Whitney, "Whittaker's cardinal function in retrospect," Math. Comp., v. 25, 1971, pp. 141-154.

3. R. A. SACK, "Comments on some quadrature formulas by F. Stenger," J. Inst. Math. Appl., v. 21, 1978, pp. 359-361.

4. F. Stenger, "Integration formulae based on the trapezoidal formula," J. Inst. Math. Appl., v. 12, 1973, pp. 103-114.

5. F. STenger, “Approximations via Whittaker's cardinal function,” J. Approx. Theory, v. 17, 1976, pp. 222-240.

6. F. Stenger, "Remarks on "Integration formulas based on the trapezoidal formula", J. Inst. Math Appl., v. 19, 1977, pp. 145-147.

7. F. STENGeR \& D. El.LIOTT, "SINC method of solution for singular integral equations," in Numerical Solution of Singular Integral Equations (A. Gerasoulis, R. Vichnevetsky, eds.), Proceedings of an IMACS International Symposium held at Lehigh University, Bethlehem, PA, USA, June 21-22, 1984, pp. 27-35.

8. J. M. WhitTAKer, "On the cardinal function of interpolation theory," Proc. Edinburgh Math. Soc. (1), v. 2, 1927, pp. 41-46

9. E. Venturino, An Analysis of Some Direct Methods for the Numerical Solution of Singular Integral Equations, Ph. D. thesis, SUNY at Stony Brook, 1984. 